\title{
Solid modeling based servo system design for a high speed micro grinding machine
}

\author{
Ruey-Jeng Lee, Kuan-Chien Chou, Shu-Hung Liu, Jia-Yush Yen* \\ Department of Mechanical Engineering, National Taiwan University, No. 1 Sec. 4 Rossevelt Rd, Taipei, Taiwan ROC
}

Received 10 November 2004; accepted 7 April 2005

Available online 24 August 2005

\begin{abstract}
The flourishing hi-tech industry development has prompted the need to carry out high performance servo design and testing without actually having to construct a prototype system. However, the complicated mechanical structure and the nonlinear effects in many of the high performance systems have made it very difficult to carry out the design without a suitable model. In recent years, the software advances has achieved very accurate dynamic simulation result. It is also possible to integrate the control synthesis with the dynamic simulation. This study examines the possibility of performing the servo design within the system design environment and then applies the result to a realistic platform model. The robust sliding mode controller is employed to deal with the nonlinear characteristics in the grinding machine. The dynamic model included the multi-degree of freedom behavior and the friction effects. The simulation results also show that the sliding mode controller is able to suppress the structure resonances and achieve high accuracy control under plant uncertainties.
\end{abstract}

(C) 2005 Elsevier Ltd. All rights reserved.

Keywords: Solid modeling based design; Sliding mode controller; Vibration suppression

\section{Introduction}

With the scientific and the technological progress are always accompanied with severe competition. The result is often a shortened product life cycle and very tight research and development schedule.

In the case of the machine tool industry, the desire is to achieve higher manufacturing accuracy with faster operation speed, and one has to accomplish the new design within a very tight schedule. The difficulty to achieve fast design is that the machine tool servo system always requires the model identification on a prototype machine and the servo tuning can be based on the system identification result. It is possible to perform parameter tuning without the system identification, but the tuning still has to be based on a prototype machine. The procedure is very expensive and time consuming to build a prototype machine.

With the advancement in the computer aided design (CAD) tool, people are now able to construct very realistic

\footnotetext{
* Corresponding author. Tel: + 8862 33662688; fax: +886223644871.

E-mail address: jyen@ntu.edu.tw (J.-Y. Yen).
}

0890-6955/\$ - see front matter (C 2005 Elsevier Ltd. All rights reserved. doi:10.1016/j.ijmachtools.2005.04.004 simulation model. While it is also possible to run dynamic simulation with these models, one starts to wonder if one can conduct servo design with these models. The ability to conduct servo design within the simulation environment would then allow the engineers to carry out the machine tool design process without actually having to construct the prototype machine and thus save tremendous amount of product development time.

This research attempts to carry out a servo design procedure within the solid modeling environment. Because all the states are available in the simulation environment, it is possible to use the popular Sliding Mode Control (SMC). The SMC has the advantage of good robustness property against system disturbances [1] and unmodeled dynamics [2]. The SMC structure can also deal with a class of system nonlinearity. To suppress the chattering phenomenon, the design also adopts boundary layer approach [3].

The control synthesis in this research is still bases on the mathematical model. The system parameters are extracted from the identification results based on the solid modeling data. The resulted controller is then applied to the more realistic dynamic simulation based on solid modeling. The dynamic simulation results show confirmation with the design purposes. 


$\begin{array}{ll}\text { Nomenclature } \\ \left(x_{i}, y_{i}\right) & \text { location of each slippers above the bearing } \\ r_{i} & \text { distance from origin to each slipper } \\ \xi_{i} & \text { angle between vector } r_{i} \text { and } x \text {-coordinate } \\ k_{t i} & \text { lateral stiffness between slipper and bearing } \\ w & \text { platform weight } \\ F_{i} & \text { the force acting on the platform } \\ b & \text { coefficient of the air resistant }\end{array}$

\section{The analysis software}

The simulation in this study involved a series of software. The computer aided design data is based on ProEngineering. The dynamic simulation and the structural modal analysis is base on Working Model [4], and the control synthesis and the system analysis is base on MATLAB, which also provides the integration test platform for overall system performance test.

The mathematical model of the system is analytically derived. The model can serve as a basis for system simulation. The system analysis is performed with MATLAB, which is a popular software package for mathematical analysis. For solid modeling, the original design is conducted with Pro-Engineering. The design data is then transferred into Working Model for dynamic simulation. The CAD model describes the detailed mechanism relationship as well as the model structure. For simplicity, the move platform is modeled for the dynamic simulation and the effect of the base is considered with as a disturbance in the MATLAB simulation. The dynamic simulation is based upon Working Model. It is capable of simulating the dynamic behavior of the moving platform including vibration, dynamic imbalance behavior like rolling, bouncing, slipping and swinging, as well as the associated forces. Working Model also provides a friendly

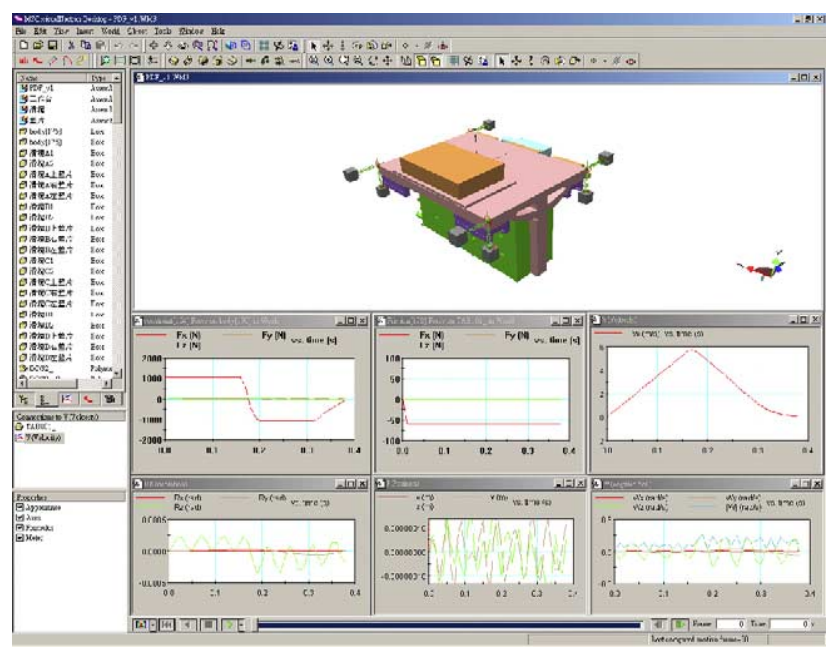

Fig. 1. The software operation interface of Working Model. $d_{\mathrm{i}} \quad$ the distance from $F_{i}$ to the centroid of the platform

$\mu_{i} \quad$ coefficient of frication

$S_{i} \quad$ the sliding surface

$K_{i} \quad$ the feedback gain of SMC controller

PK1V the gain of PDF controller

PK2V the gain of PDF controller

\section{System modeling}

The controller synthesis is based on an analytical model. The analytical model can then be combined with the structural consideration to take into account the structural resonances.

Because the main moving part is the moving platform, a model is derived to describe the platform dynamics. Proper constraints are introduced to simulate the actual operation as shown in Fig. 2. The acting force is introduced by two linear motors under the platform. Their forces are denoted by F1 and F2. There are four linear bearings to support the moving platform. Their effect is modeled by eight very stiff springs moving with the platform. The spring constant is taken as the bearing stiffness. The orientation of the mathematical model is depicted in Fig. 3.

\subsection{Lateral forces}

We build a $X-Y$ coordinate and the origin is located in the centroid of the platform. The geometric relationship showed in Fig. 3 [4]:

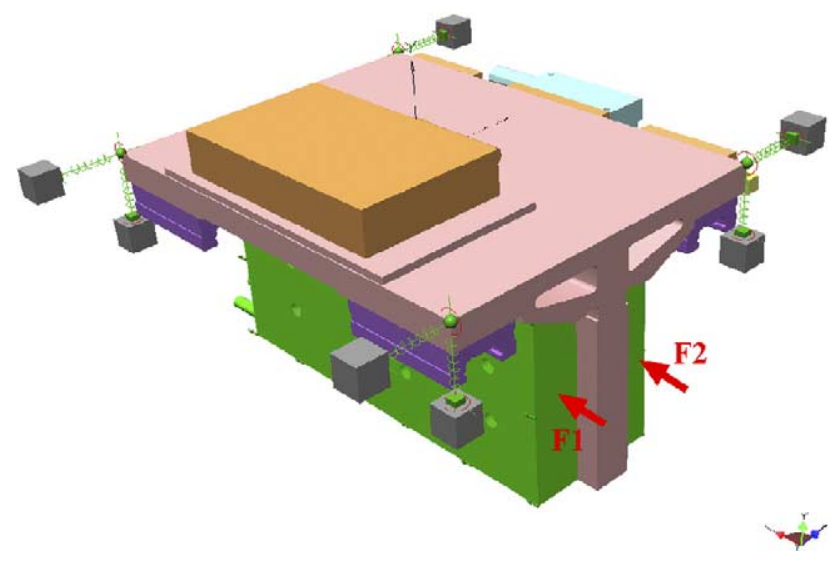

Fig. 2. Model sketch map of the platform. 


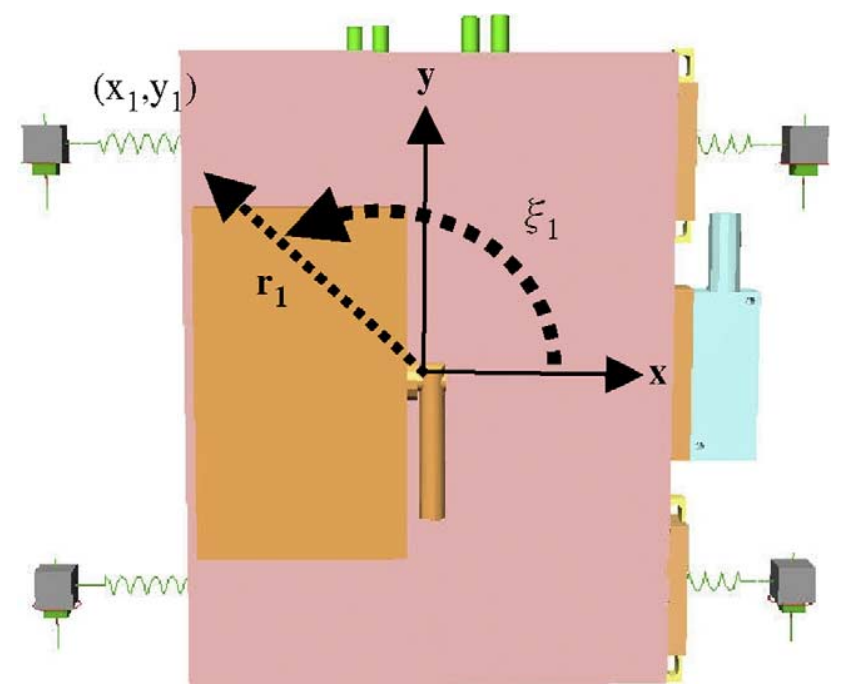

Fig. 3. Schematics of the coordinate system.

$\left\{\begin{array}{l}x_{1}=r_{1} \cdot \cos \xi_{1} \\ y_{1}=r_{1} \cdot \sin \xi_{1}\end{array}\right.$.

Taking the differential operation one obtain

$\left\{\begin{array}{l}\mathrm{d} x_{1}=-r_{1} \cdot \sin \xi_{1} \cdot \mathrm{d} \xi_{1} \\ \mathrm{~d} y_{1}=r_{1} \cdot \cos \xi_{1} \cdot \mathrm{d} \xi_{1}\end{array}\right.$.

Similarly,

$$
\begin{aligned}
& \left\{\begin{array} { l } 
{ x _ { 2 } = r _ { 2 } \cdot \operatorname { c o s } \xi _ { 2 } } \\
{ y _ { 2 } = r _ { 2 } \cdot \operatorname { s i n } \xi _ { 2 } }
\end{array} \rightarrow \left\{\begin{array}{l}
d x_{2}=-r_{2} \cdot \sin \xi_{2} \cdot d \xi_{2} \\
d y_{2}=r_{2} \cdot \cos \xi_{2} \cdot d \xi_{2}
\end{array}\right.\right. \\
& \left\{\begin{array} { l } 
{ x _ { 3 } = r _ { 3 } \cdot \operatorname { c o s } \xi _ { 3 } } \\
{ y _ { 3 } = r _ { 3 } \cdot \operatorname { s i n } \xi _ { 3 } }
\end{array} \rightarrow \left\{\begin{array}{l}
d x_{3}=-r_{3} \cdot \sin \xi_{3} \cdot d \xi_{3} \\
d y_{3}=r_{3} \cdot \cos \xi_{3} \cdot d \xi_{3}
\end{array}\right.\right. \\
& \left\{\begin{array} { l } 
{ x _ { 4 } = r _ { 4 } \cdot \operatorname { c o s } \xi _ { 4 } } \\
{ y _ { 4 } = r _ { 4 } \cdot \operatorname { s i n } \xi _ { 4 } }
\end{array} \rightarrow \left\{\begin{array}{l}
d x_{4}=-r_{4} \cdot \sin \xi_{4} \cdot d \xi_{4} \\
d y_{4}=r_{4} \cdot \cos \xi_{4} \cdot d \xi_{4}
\end{array}\right.\right.
\end{aligned}
$$

because $\mathrm{d} \xi_{1}=\mathrm{d} \xi_{2}=\mathrm{d} \xi_{3}=\mathrm{d} \xi_{4}$, it is possible to represent all of them by $\mathrm{d} \xi$.

The effect of the linear bearing forces can now be calculated as

$$
\begin{aligned}
& \overline{F_{t 1}}=-k_{t 1} \cdot \mathrm{d} x_{1}=-k_{t 1} \cdot-r_{1} \cdot \sin \xi_{1} \cdot \xi=k_{t 1} \cdot r_{1} \sin \xi_{1} \cdot \xi \\
& \overline{F_{t 2}}=-k_{t 2} \cdot \mathrm{d} x_{2}=-k_{t 2} \cdot-r_{2} \cdot \sin \xi_{2} \cdot \xi=k_{t 2} \cdot r_{2} \sin \xi_{2} \cdot \xi \\
& \overline{F_{t 3}}=-k_{t 3} \cdot \mathrm{d} x_{3}=-k_{t 3} \cdot-r_{3} \cdot \sin \xi_{3} \cdot \xi=k_{t 3} \cdot r_{3} \sin \xi_{3} \cdot \xi \\
& \overline{F_{t 4}}=-k_{t 4} \cdot \mathrm{d} x_{4}=-k_{t 4} \cdot-r_{4} \cdot \sin \xi_{4} \cdot \xi=k_{t 4} \cdot r_{4} \sin \xi_{4} \cdot \xi .
\end{aligned}
$$

Through the same process, the resulted moment on the platform would then become: assume $k_{t 1}=k_{t 2}=k_{t 3}=$ $k_{t 4}=k_{t}$,

Moment:

$M=k_{e} \cdot \xi$ where

$$
\begin{aligned}
k_{e}= & -k_{t} \cdot\left(r_{1} \sin \xi_{1} \cdot y_{1}+r_{2} \sin \xi_{2} \cdot y_{2}+r_{3} \sin \xi_{3} \cdot y_{3}\right. \\
& \left.+r_{4} \sin \xi_{4} \cdot y_{4}\right)
\end{aligned}
$$

The frictional force in the $y$ direction is then:

$F_{r}=k_{r} \cdot|\xi| \cdot \operatorname{sgn}(\dot{y})$

where

$k_{r}=-k_{t}\left(r_{1} \sin \xi_{1}-r_{2} \sin \xi_{2}-r_{3} \sin \xi_{3}+r_{4} \sin \xi_{4}\right) \cdot \mu_{s}$

The moment caused by the frictional force in the $y$ direction then becomes:

$M_{r}=k_{m}|\xi| \operatorname{sgn}(\dot{y})$

where

$$
\begin{aligned}
k_{m}= & \left(-x_{1} r_{1} \sin \xi_{1}+x_{2} r_{2} \sin \xi_{2}+x_{3} r_{3} \sin \xi_{3}\right. \\
& \left.-x_{4} r_{4} \sin \xi_{4}\right) k_{t} \mu_{s}
\end{aligned}
$$

Collecting all the forces and substitute them into the dynamic relationship

$\sum \vec{F}=m \ddot{x}, \quad \sum \vec{M}=I \ddot{\theta}$

one now obtain the system dynamics in the $y$ and the $z$ directions

$$
\begin{gathered}
F_{1}+F_{2}+F_{r}-W \mu_{s} \operatorname{sgn}(\dot{y})-b \dot{y}=m \ddot{y} \\
-F_{1} d_{1}+F_{2} d_{2}+M+M_{r}=I_{z} \ddot{\xi}
\end{gathered}
$$

where $W$ is the platform weight, $b$ is the coefficient of the air resistant, $d_{1}$ is the distance from $F_{1}$ to the centroid of the platform, $d_{2}$ is the distance from $F_{2}$ to the platform centroid.

Rearranging, one obtain the system representation of the moving platform as:

$$
\begin{aligned}
{\left[\begin{array}{c}
\dot{y} \\
\ddot{y} \\
\dot{\xi} \\
\ddot{\xi}
\end{array}\right]=} & {\left[\begin{array}{cccc}
0 & 1 & 0 & 0 \\
0 & -\frac{b}{m} & \frac{k_{r} \cdot \operatorname{sgn}(\dot{y} \xi)}{m} & 0 \\
0 & 0 & 0 & 1 \\
0 & 0 & \frac{k_{e}+k_{m} \cdot \operatorname{sgn}(\dot{y} \xi)}{I z} & 0
\end{array}\right]\left[\begin{array}{c}
y \\
\dot{y} \\
\xi \\
\dot{\xi}
\end{array}\right] } \\
& +\left[\begin{array}{cc}
0 & 0 \\
\frac{1}{m} & \frac{1}{m} \\
0 & 0 \\
-\frac{d_{1}}{I z} & \frac{d_{2}}{I z}
\end{array}\right]\left[\begin{array}{c}
F_{1} \\
F_{2}
\end{array}\right]-\left[\begin{array}{c}
0 \\
\frac{W \mu_{s} \operatorname{sgn}(\dot{y})}{m} \\
0 \\
0
\end{array}\right]
\end{aligned}
$$

Notice that the friction disturbance does not enter the system through the input channel. Fortunately, it is possible 
to rearrange the system expression again to obtain:

$$
\begin{aligned}
{\left[\begin{array}{c}
\dot{y} \\
\ddot{y} \\
\dot{\xi} \\
\ddot{\xi}
\end{array}\right]=} & {\left[\begin{array}{cccc}
0 & 1 & 0 & 0 \\
0 & -\frac{b}{m} & \frac{k_{r} \cdot \operatorname{sgn}(\dot{y} \xi)}{m} & 0 \\
0 & 0 & 0 & 1 \\
0 & 0 & \frac{k_{e}+k_{m} \cdot \operatorname{sgn}(\dot{y} \xi)}{I z} & 0
\end{array}\right]\left[\begin{array}{l}
y \\
\dot{y} \\
\xi \\
\dot{\xi}
\end{array}\right] } \\
& +\left[\begin{array}{cc}
0 & 0 \\
\frac{1}{m} & \frac{1}{m} \\
0 & 0 \\
-\frac{d_{1}}{I z} & \frac{d_{2}}{I z}
\end{array}\right]\left[\begin{array}{l}
F_{1}-\frac{d_{2}}{d_{1}+d_{2}} W \mu_{s} \operatorname{sgn}(\dot{y}) \\
F_{2}-\frac{d_{1}}{d_{1}+d_{2}} W \mu_{s} \operatorname{sgn}(\dot{y})
\end{array}\right]
\end{aligned}
$$

We define

$A=\left[\begin{array}{cccc}0 & 1 & 0 & 0 \\ 0 & -\frac{b}{m} & \frac{k_{r} \cdot \operatorname{sgn}(\dot{y} \xi)}{m} & 0 \\ 0 & 0 & 0 & 1 \\ 0 & 0 & \frac{k_{e}+k_{m} \cdot \operatorname{sgn}(\dot{y} \xi)}{I z} & 0\end{array}\right]$ and $B=\left[\begin{array}{cc}0 & 0 \\ \frac{1}{m} & \frac{1}{m} \\ 0 & 0 \\ -\frac{d_{1}}{I z} & \frac{d_{2}}{I z}\end{array}\right]$
Notice that the friction disturbance is now a matched disturbance.

\section{Design and simulation of sliding mode controller}

Introducing the control [5]

$u=-(H B)^{-1}(H A X+K \cdot \operatorname{sgn}(S))$

where we define $K=\left[\begin{array}{ll}K_{1} & K_{2}\end{array}\right]$ is the feedback gain of SMC controller,

$S=\left[\begin{array}{l}S_{1} \\ S_{2}\end{array}\right]$

is the sliding surface, $S=H X$ and $H$ is chosen to make $[A-$ $\left.B(H B)^{-1} H A\right]$ Hurwitz. Substitute $u$ into the Lyapunov function $V=S^{\mathrm{T}} S$ [6], it is easy to show that the system will converge to the sliding surface.

$$
\begin{aligned}
\dot{V} & =2 S^{T} \dot{S}=2 S^{T}(H A X+H B u) \\
& =-2\left[K_{1} S_{1} \operatorname{sgn}\left(S_{1}\right)+K_{2} S_{2} \operatorname{sgn}\left(S_{2}\right)\right]
\end{aligned}
$$

Setting $K_{1}$ and $K_{2}$ positive, one gets $V>0$, and $\dot{V}<0$. The system states will converge to a sliding surface by a suitably designed input. If the sliding surface is stable, then the system will converge to an equilibrium point.

The controller is then implemented with MATLAB as shown in Fig. 4. To avoid high frequency chattering,

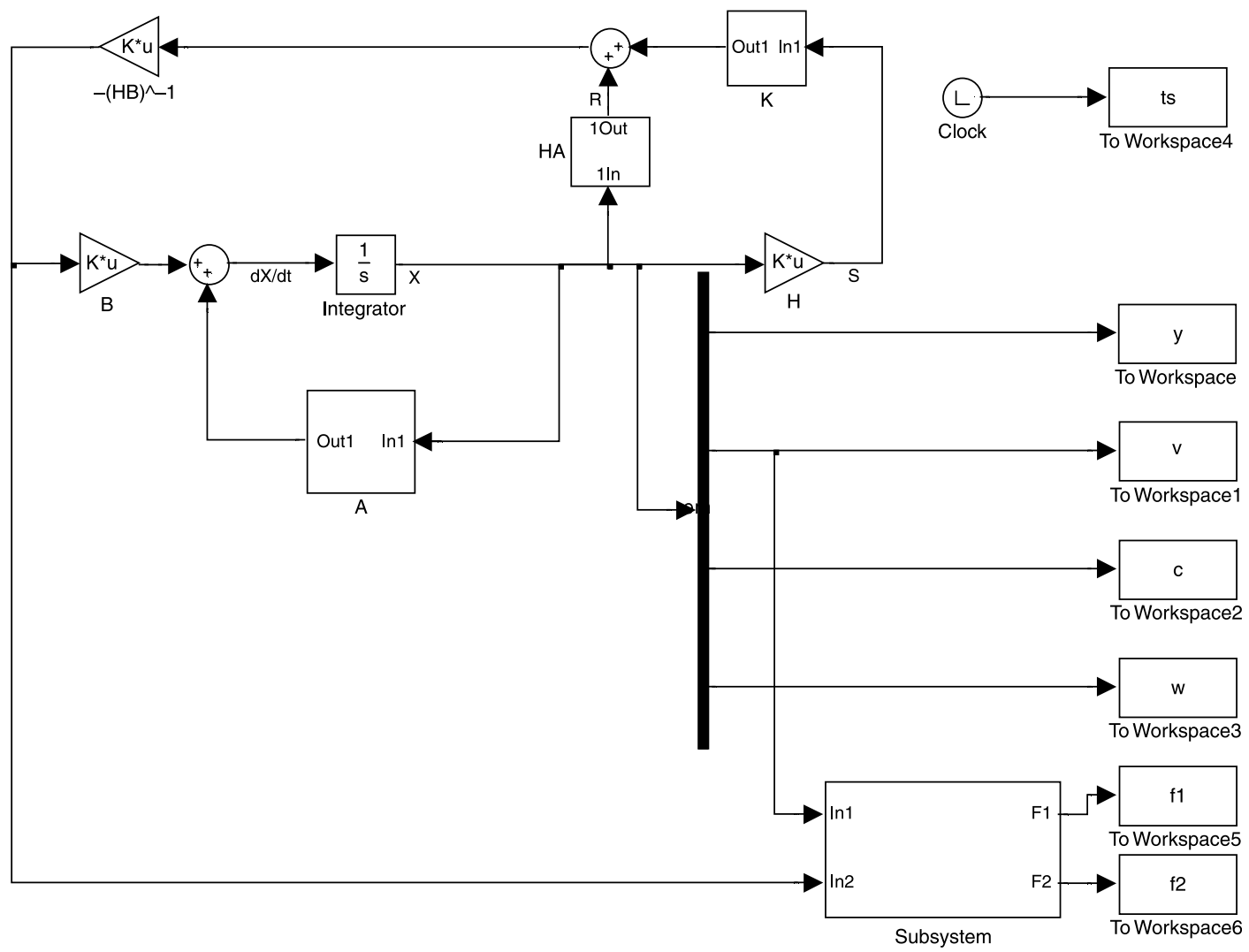

Fig. 4. The scheme of sliding mode control system. 


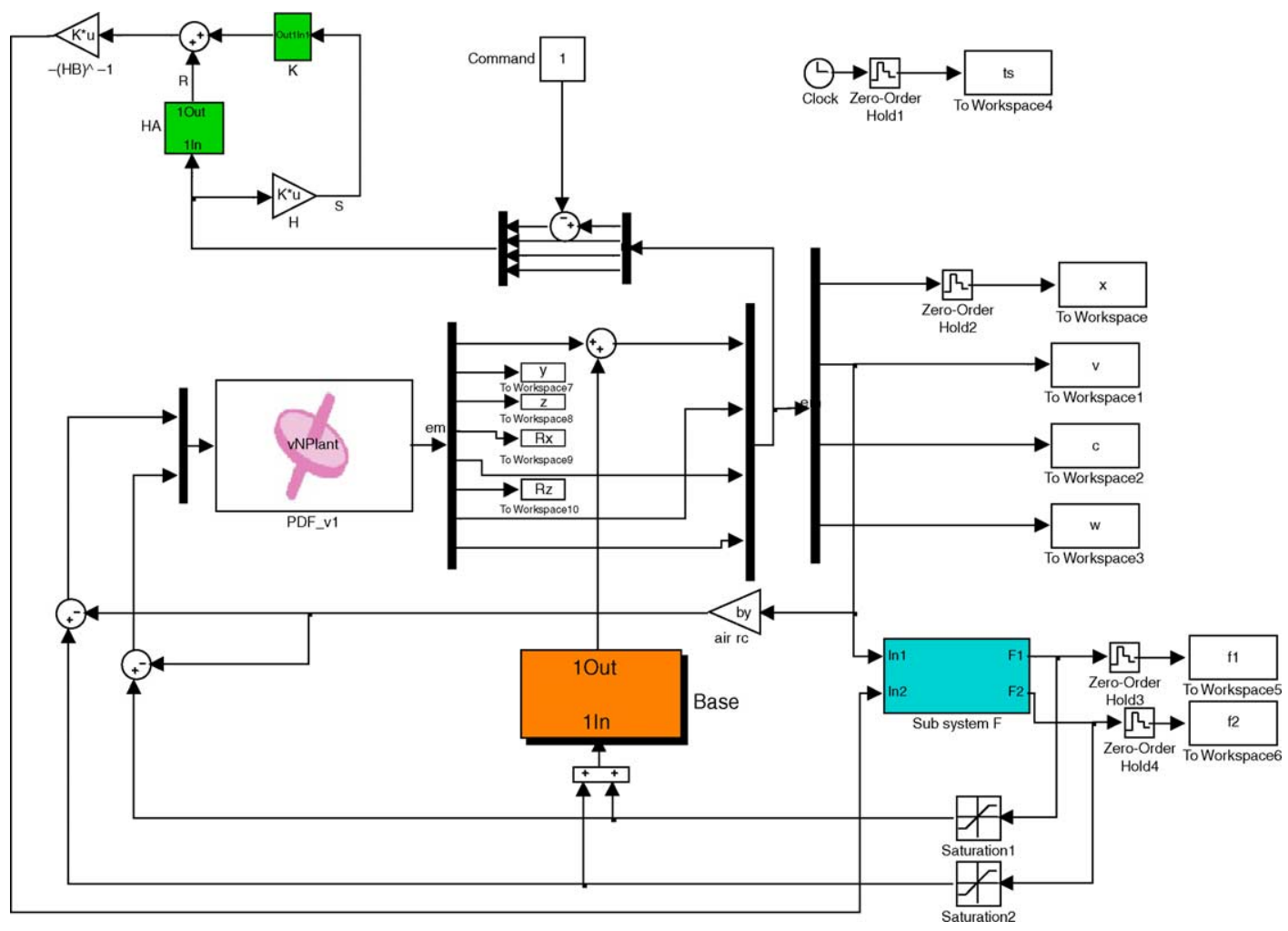

Fig. 5. Overview of the control scheme.

the design also includes a boundary layer around the sliding surface. A saturation function in the simulation replaces the sigmoid block in the simulation.

There are two design parameters in the SMC setup: the sliding surface $H$ and the feedback gain $K$. Different values of $H$ and $K$ 's are tested to find the best performance.

\section{Integrated simulation and result analysis}

This section shows the test results from the integration of Working Model with MATLAB.

The structural resonances from the solid modeling are introduced into the system at this stage. To be more complete, the simulation also includes the air resistance effect. The simulation environment is shown in Fig. 5. The moving platform constitutes the vNMODEL block in the schematics. The simulation result can be viewed from the operational interface in Fig. 1.

\section{Solid modeling based simulation}

A simulation is first carried out with the same initial conditions to the analytical model and the solid modeling models. The difference is found out to be quite small.
(The difference mainly arises from the joining of the resonance mode and air resistance factor.)

The control tests are first carried out with different feedback gain values: $K_{1}=32,64,50,40$ for analysis. The control with $K_{1}=32$ and 40 yield better performances. Comparing the results with $K_{1}=32$ and 40 , one finds that the system converges more quickly at $K_{1}=40$. This then becomes the setup for the following analysis.

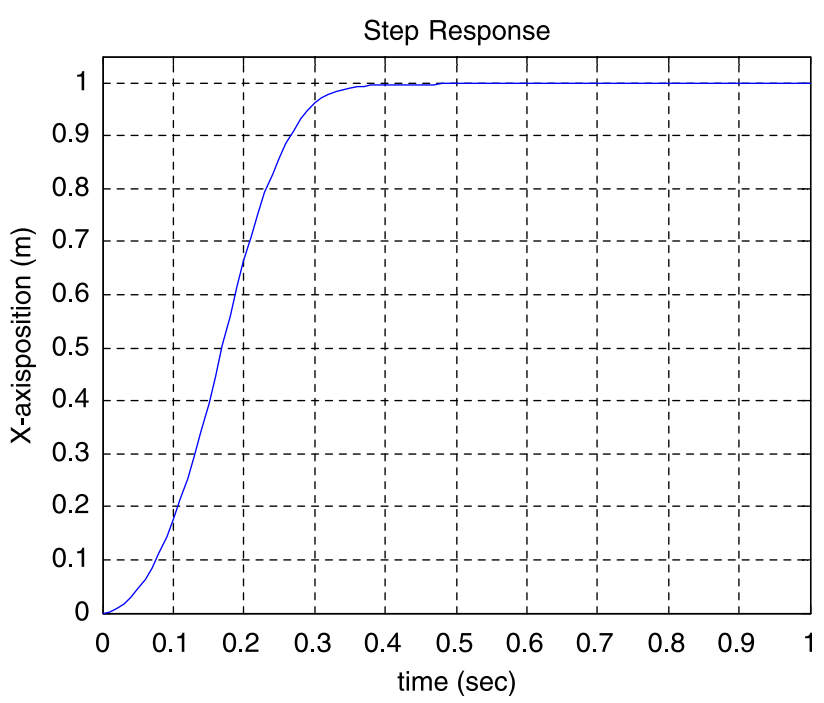

Fig. 6. Centroid to time in $x$ direction. 


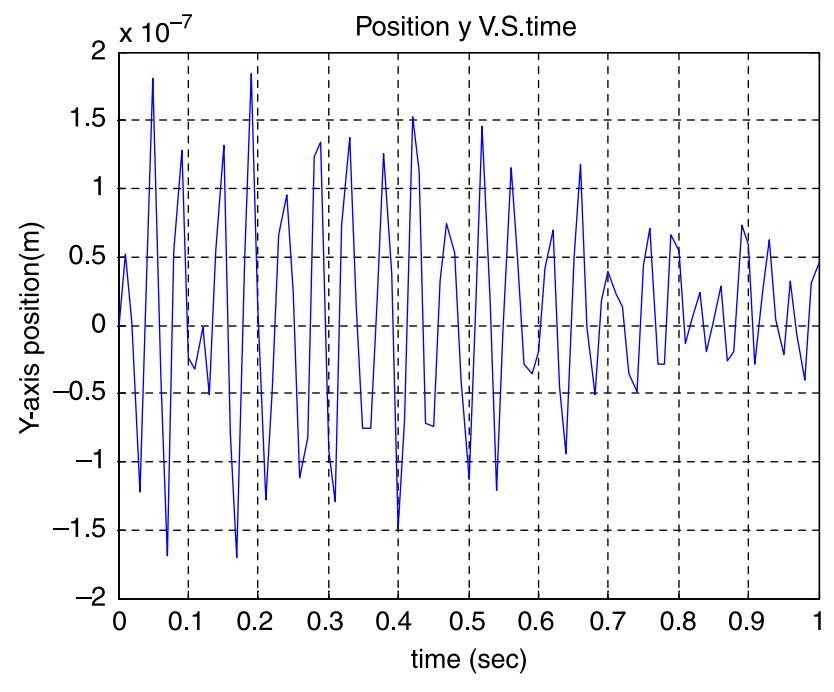

Fig. 7. Centroid to time in $y$ direction.

The simulation results are shown in Figs. 6-9. The settling time is less than $400 \mathrm{~ms}$. In the lateral direction, one observes a sustained vibration of amplitude less than $\pm 2 \times$ $10^{-6} \mathrm{~m}$. In the rotational directions as shown in the figures in Fig. 9, there are less than $10^{-4}$ rad vibration in the $x, y$, and $z$ direction. The resulted displacement from these vibrations would be less than one micron, and the system converges to steady state within $400 \mathrm{~ms}$ Fig. 10.

\section{Robustness verification}

In verifying the robustness, the simulation uses different loading to the platform and obtain very similar responses with all trial conditions (Fig. 11). Similarly, in Fig. 12, one obtains similar responses with different gain values. This verifies the system robustness characteristics.

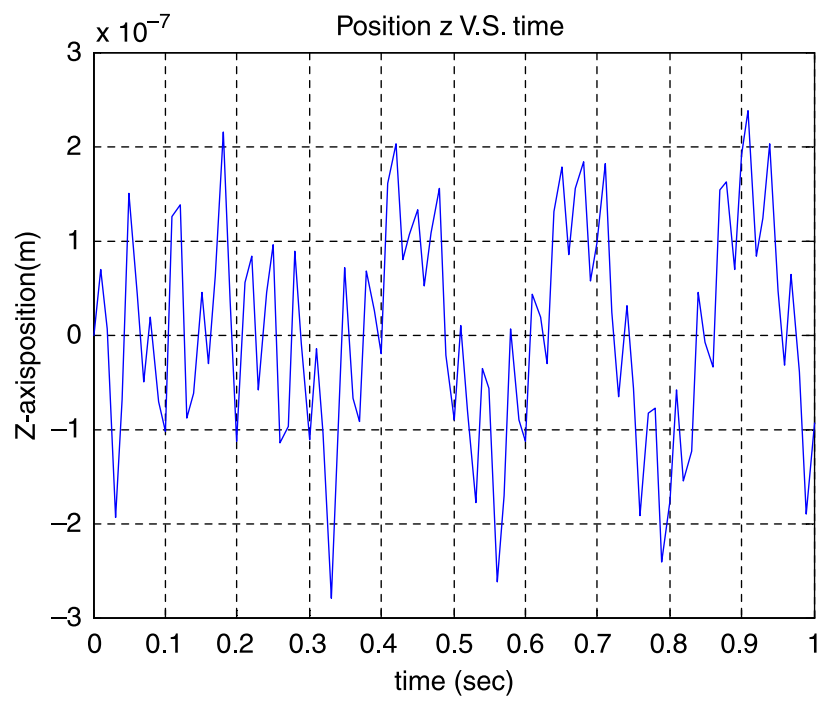

Fig. 8. Centroid to time in $z$ direction.
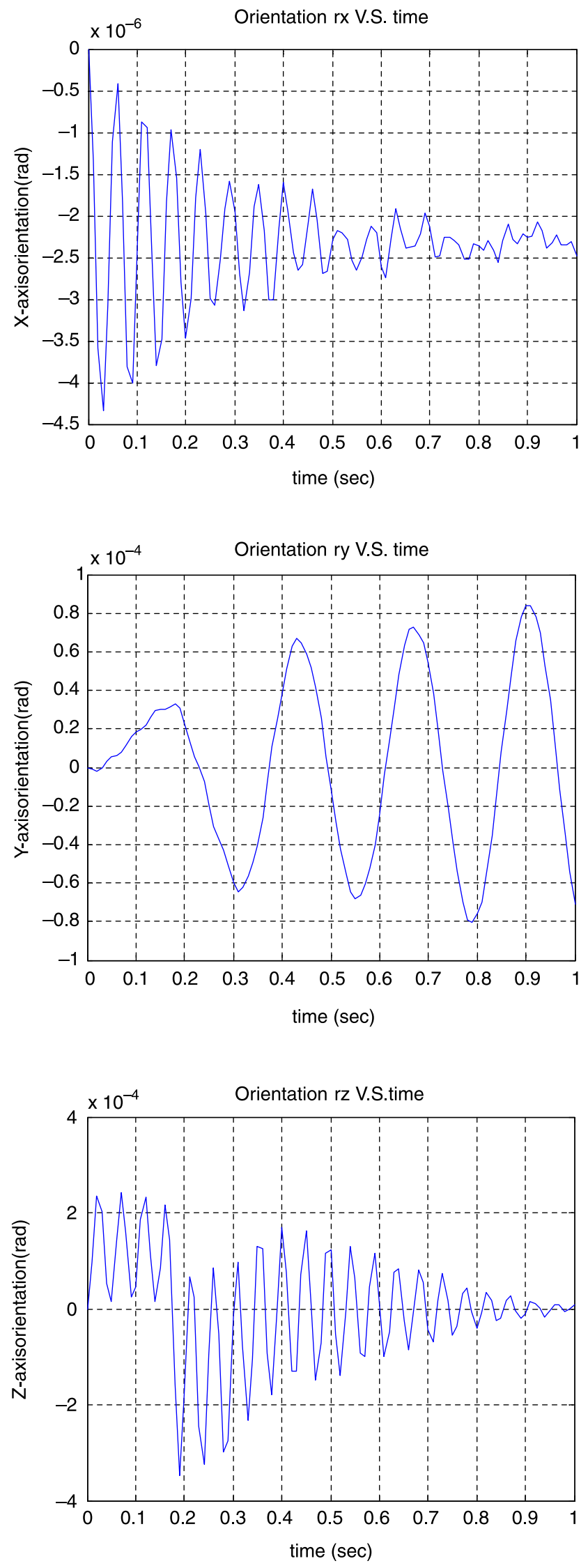

Fig. 9. Angle displacement to time. 


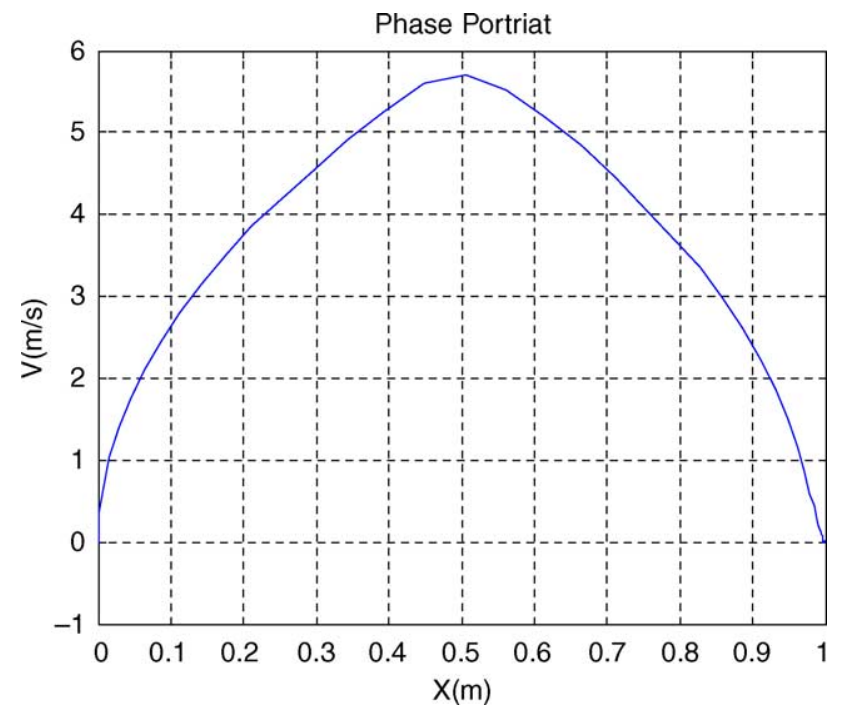

Fig. 10. Phase Portrait of $V$ to $x$.

\section{Reducing SMC to PDF control}

Because the actual commercial controllers use PDF control (Pseudo-Derivative Feedback Controller), it is desirable to reduce the SMC controller into a PDF format (Fig. 13) for practical implementations. The process is a linearization and a model reduction to reduce the controller into the PDF structure.

$C(s)=\frac{-1290 s-13,800}{s}$

Comparing with the PDF controller, the parameters in the FANUC controller (Fig. 14) would be set to PK1V $=13800$, $\mathrm{PK} 2 \mathrm{~V}=-1290$. The solid modeling simulation results in a vibrating trajectory around the equilibrium point (Fig. 15). It is suspected that the vibration is caused by the high gains, therefore, a proportional reduction of the gains to

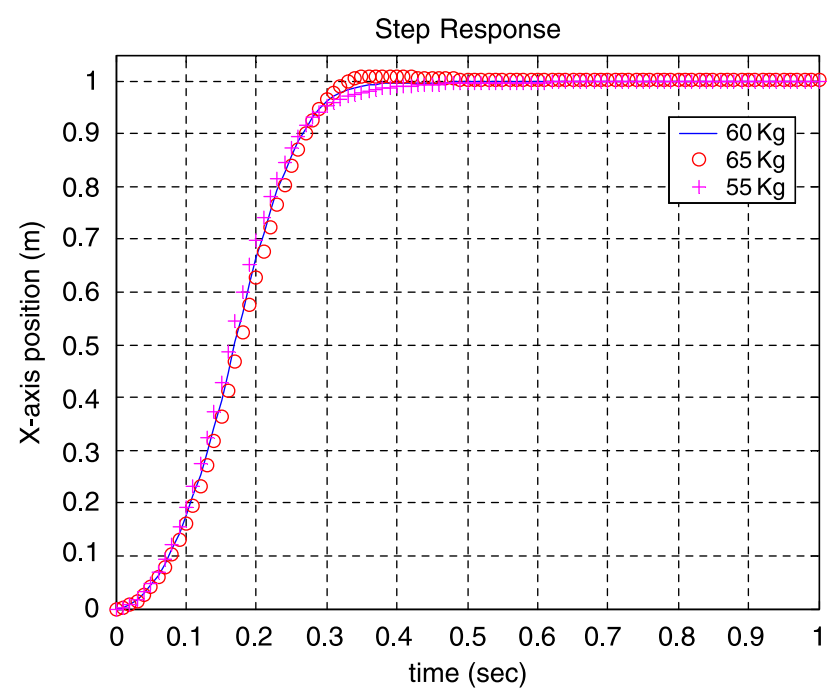

Fig. 11. Displacement to time (adjust the loading).

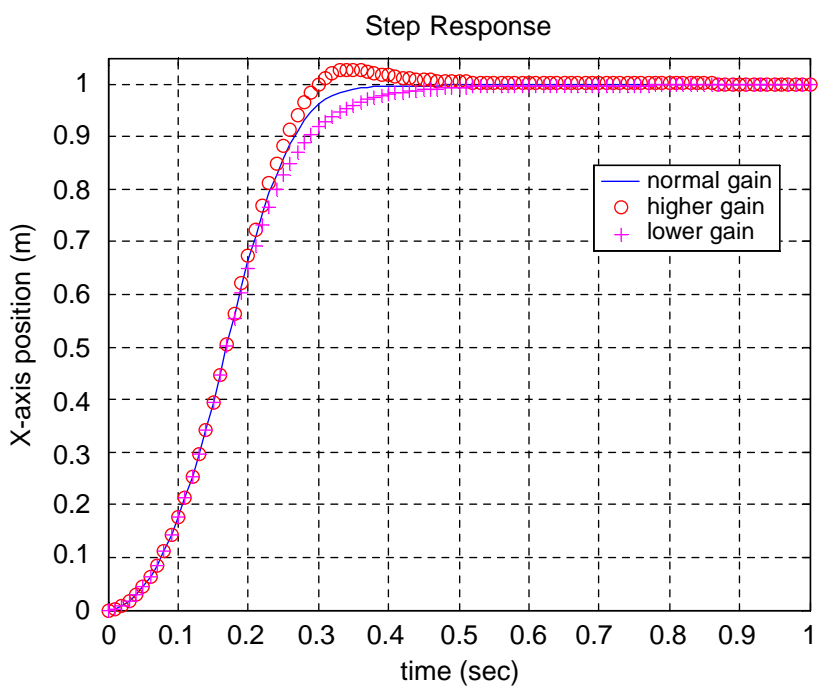

Fig. 12. Displacement to time with different gains.

$\mathrm{PK} 1 \mathrm{~V}=1600$ and $\mathrm{PK} 2 \mathrm{~V}=-149.56$ yields a smooth control result (Fig. 16).

The new parameters in the PDF controller make the platform dynamics converges in about $0.2 \mathrm{~s}$. The sustained vibration is kept under $\pm 2 \times 10^{-7} \mathrm{~m}$, and the changes in the orientations of $x, y, z$ directions are all kept under $10^{-}$ ${ }^{6} \mathrm{rad}$ (Figs. 17-19). The resulted grinding processing error can then be controlled at a level lower than one micron. Compare these results with the control result of SMC, it is interesting to observe that the reduced PDF controller leads to better performance than the original SMC. The is achieved by sacrificing robustness.

As a comparison, Fig. 20 shows the simulation of a $30 \mathrm{~mm}$ cyclic motion of the three controllers: PDF controller, reduced SMC to PDF, reduced SMC to PDF on solid modeling. Dotted line in Fig. 20 is the reduced SMC response, dash-point line is the PDF controller applied to solid modeling, and solid line represents the result with the reduced SMC to PDF controller on the solid modeling. The SMC response exhibits chattering phenomenon through high gain effect. The reduced SMC achieves better performance.

\section{Conclusions}

This paper described a procedure to conduct realistic machine tool servo design within the simulation environment. The servo control synthesis is based on the analytical model, but with the model parameters extracted from the solid modeling simulation. The controller tuning is then performed with the dynamic simulation environment with the CAD model. The CAD model reflects very realistic system dynamic behavior. The resulted controller is directly applicable to the actual machine. Thus, the process reduces (or eliminates) the need for an expensive prototype. 


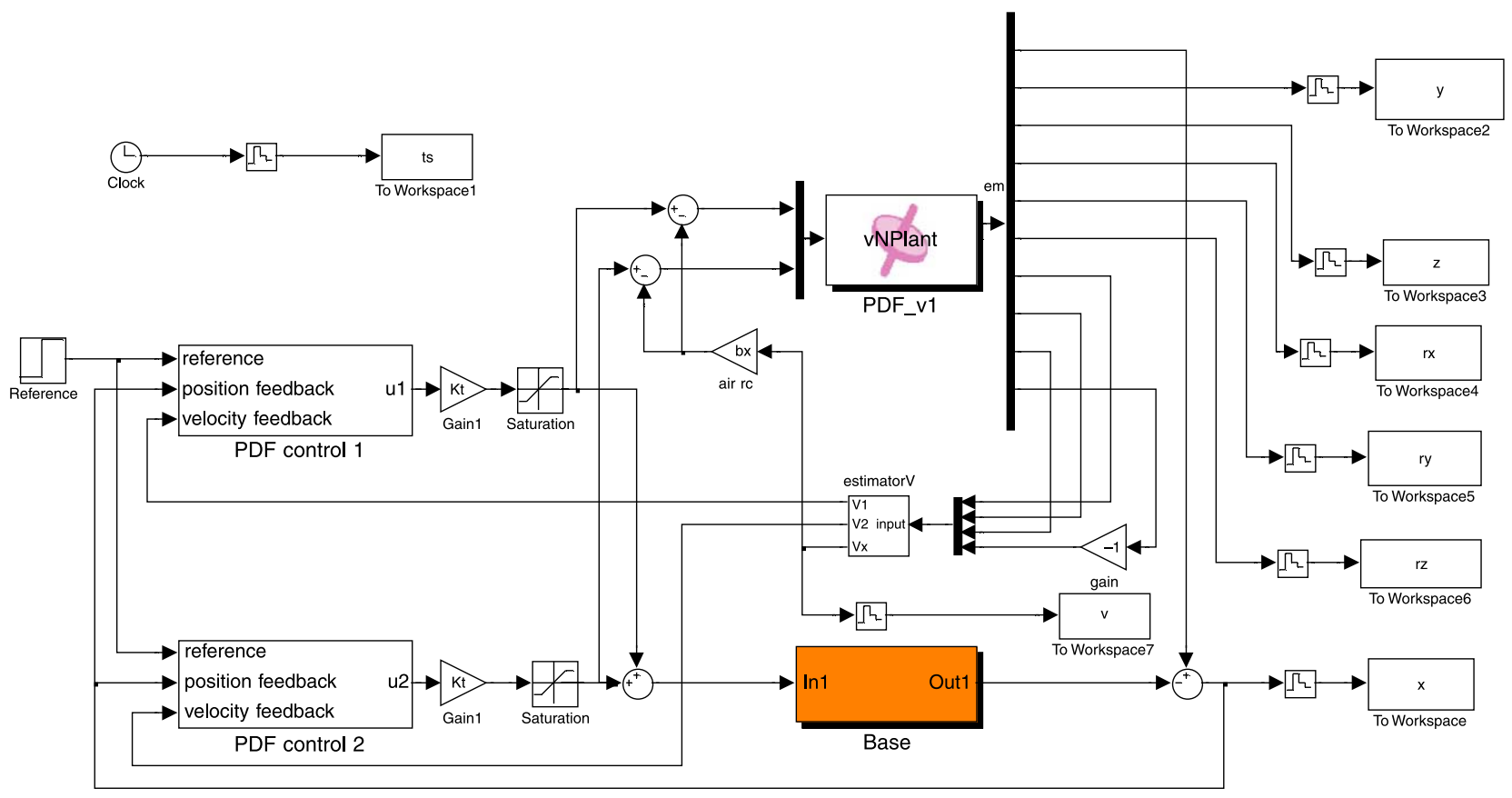

Fig. 13. Overview of PDF control scheme.

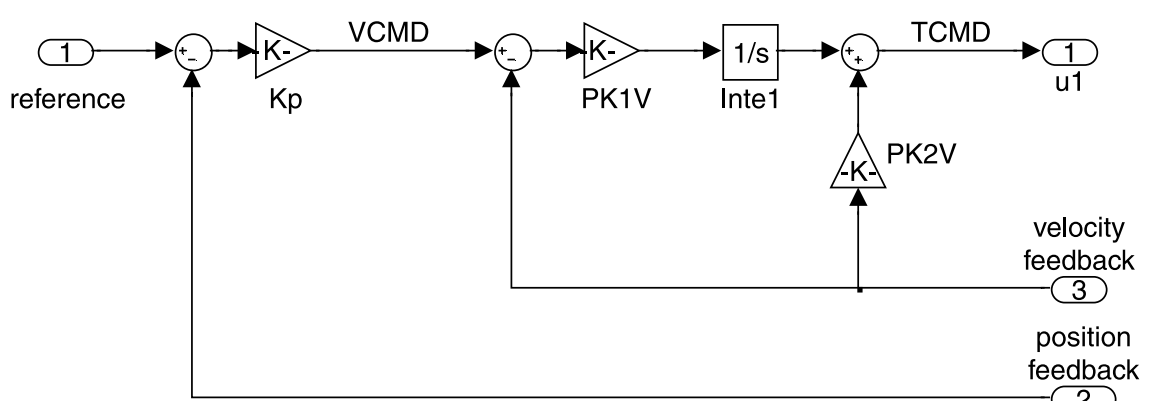

Fig. 14. PDF structure in the FANUC controller.

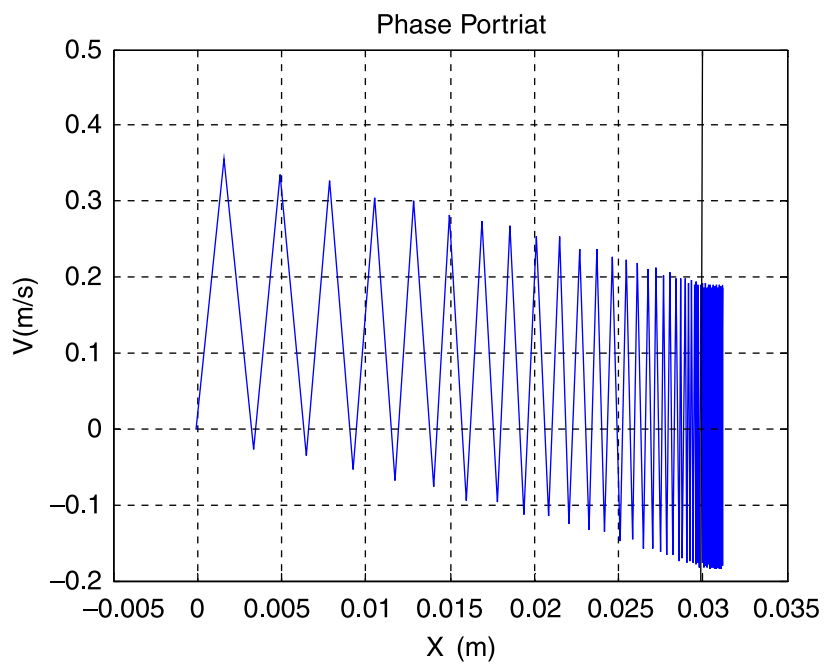

Fig. 15. Phase Portrait $(\mathrm{PK} 1 \mathrm{~V}=13800, \mathrm{PK} 2 \mathrm{~V}=-1290)$.

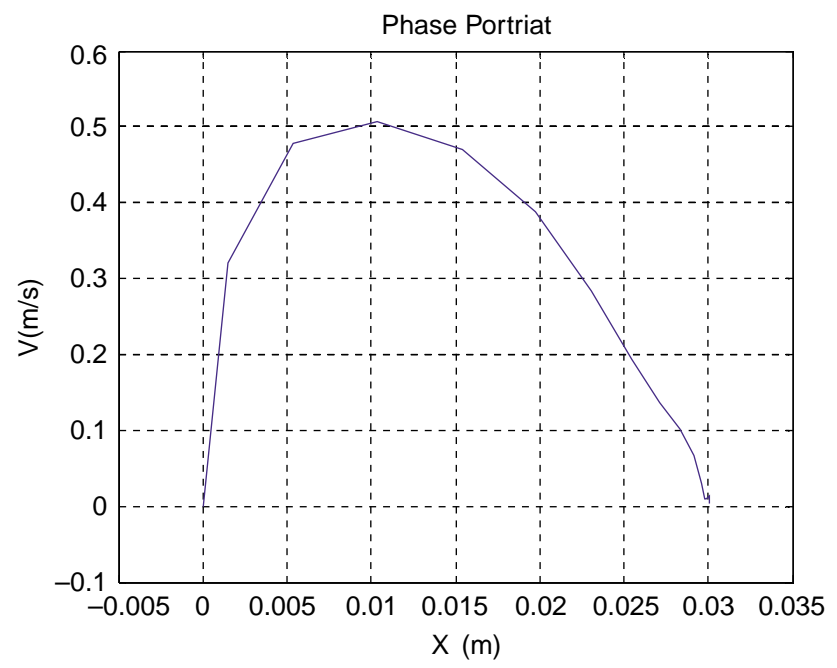

Fig. 16. Phase Portrait (PK1V=1600, PK2V $=-149.56)$. 


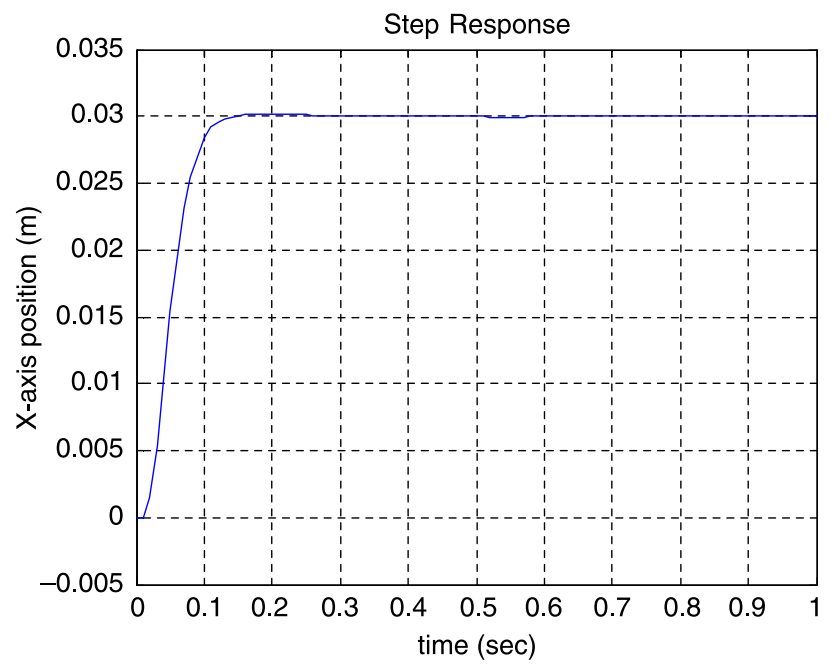

Fig. 17. Step response.
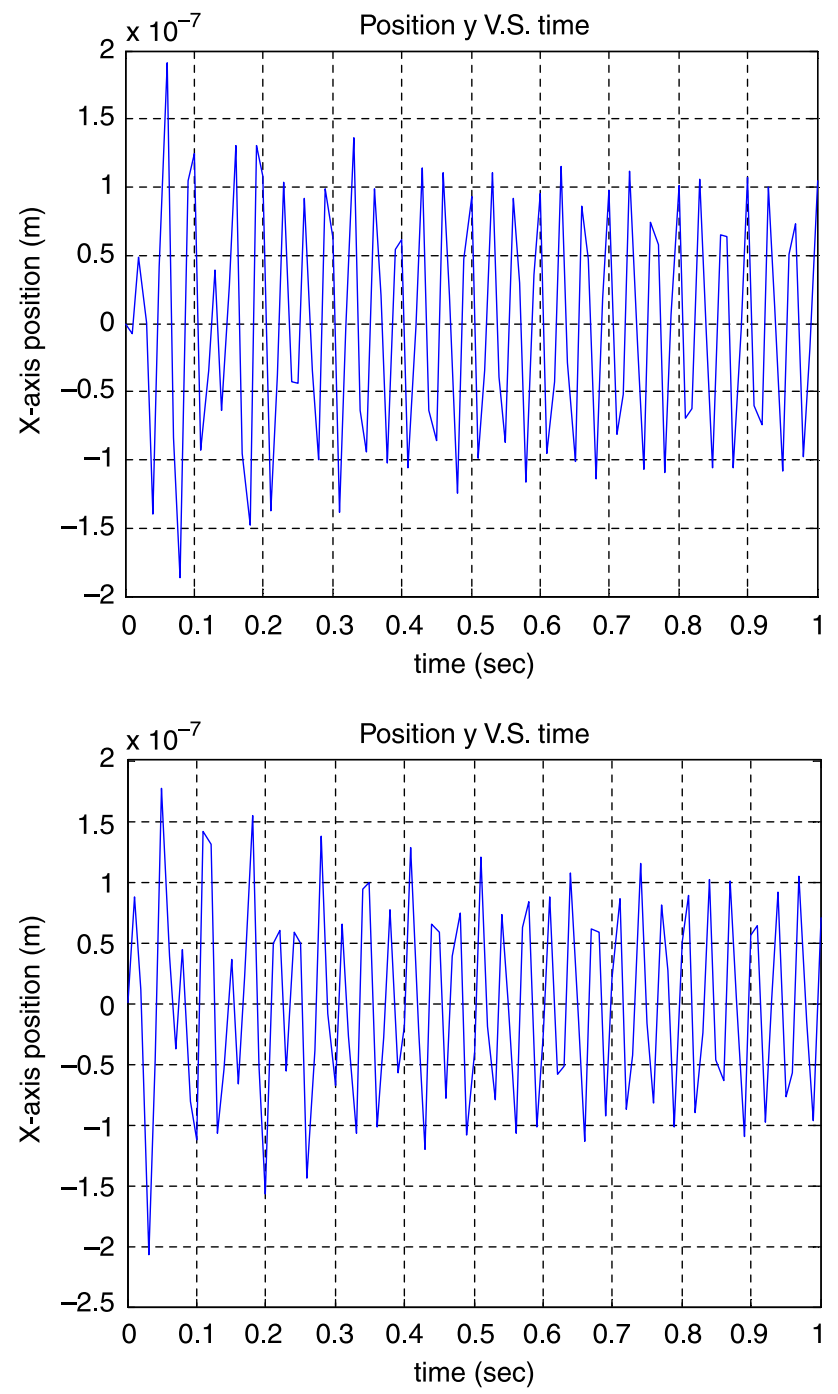

Fig. 18. Centroid to time in $x, y$ direction.
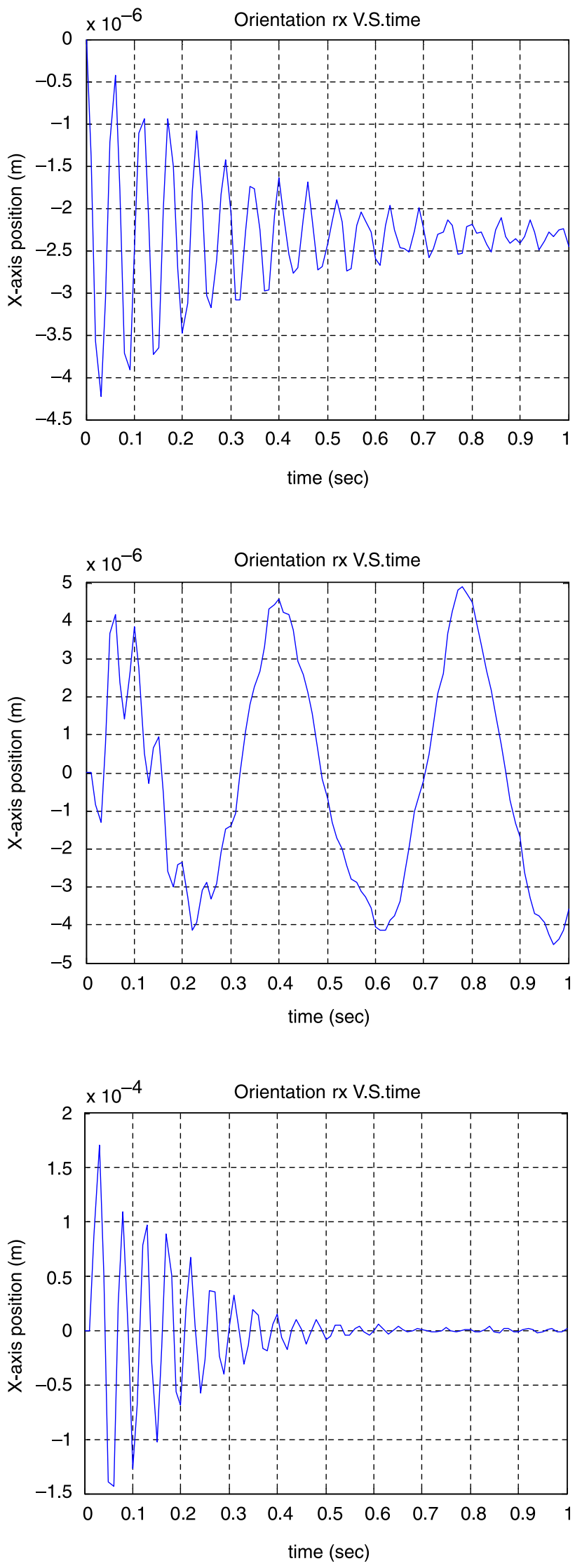

Fig. 19. Orientation to time in $x, y, z$ direction. 


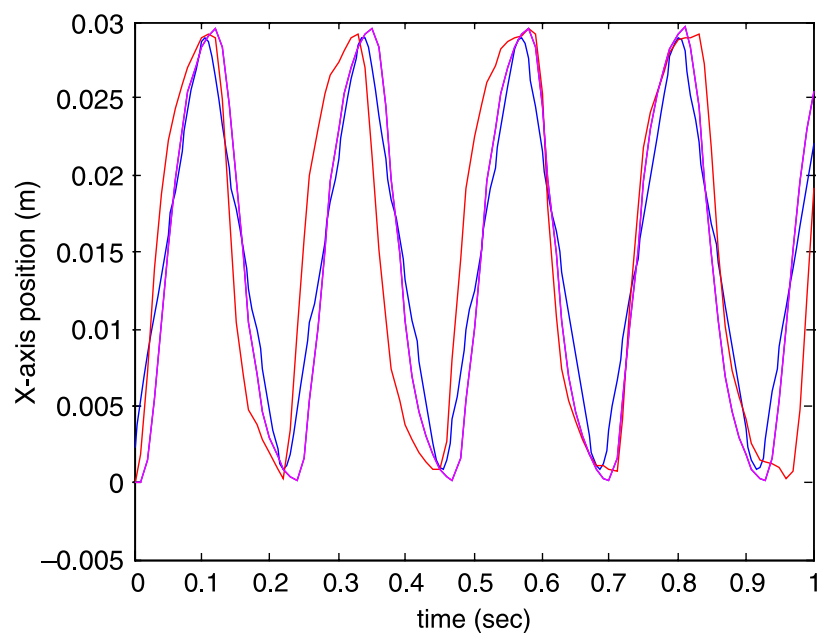

Fig. 20. Picture to time of displacement.

This process also allows the designer to examine particular control behavior with the convenience of the simulation environment.

\section{Acknowledgements}

This work is supported by the Chevalier Machinery Inc., Taichung, Taiwan under contract no. 91-SA59.

\section{References}

[1] I. Haskara, U. Uzguner, V. Utkin, Variable Structure Control for Uncertain Sampled Data Systems, Proceedings of the 36th IEEE Conference on Decision and Control, vol. 4, 1997 p. 3226-31.

[2] J. Spurgeon, R. Davies, A Nonlinear Design Approach for Sliding Mode Control Systems, Proceedings of the 32nd IEEE Conference on Decision and Control, vol. 2, 1993 p. 1440-45.

[3] J.J. Slotine, S.S. Sastry, Tracking control of nonlinear systems using sliding surfaces with application to robot manipulators, Int. J. Control 38 (2) (1983) 465-492.

[4] Tsair-Jinn Cheng, A High Speed Machine Tool Servo Design-A System Approach, Master's Thesis, Department of Mechanical Engineering, National Taiwan University, 2000.

[5] Tsang-Li Tai, On the Design of Discrete-time Sliding Mode Controller for a Class of Systems and its Application a Dissertation Submitted in Partial Fulfillment of the Requirements for the Degree of Doctor of Philosophy in Power Mechanical Engineer, National Tsing Hua University, 2002.

[6] M. Rodic, K. Jezernik, Speed-sensorless sliding-mode torque control of an induction motor, IEEE Trans. Ind. Electron. 49 (1) (2002) 87-95.

\section{Further Reading}

[1] MSC.visualNastran Desktop, MSC.visualNastran Desktop TM, MSC Software, 2001.

[2] Zhao-Ming Huang, Nonlinear Controller Design of Repulsive Maglev Suspension Guiding System, Thesis for the Doctorate, Department of Mechanical Engineering, National Taiwan University, 1999. 